\title{
Nomogram Predicting Overall Survival After Surgical Resection For Retroperitoneal Leiomyosarcoma Patients
}

\section{Aobo Zhuang}

Fudan University

Hanxing Tong

Fudan University

Yuan Fang

Fudan University

Lijie Ma

Fudan University

Weiqi Lu

Fudan University

Yong Zhang ( $\sim$ 13681971072@163.com )

Fudan University

\section{Research Article}

Keywords: asian, retroperitoneal leiomyosarcoma, prognosis, nomogram, overall survival (OS)

Posted Date: October 27th, 2021

DOI: https://doi.org/10.21203/rs.3.rs-1009507/v1

License: (1) This work is licensed under a Creative Commons Attribution 4.0 International License. Read Full License 


\section{Abstract}

Aim: To develop a survival nomogram for patients with retroperitoneal leiomyosarcoma (RLMS) after surgery.

Methods: 118 patients with RLMS after surgical resection at the General Surgery Department, Shanghai Public Health Clinical Center, Fudan University were retrospectively analyzed. The nomogram was constructed based on COX regression model and discrimination was assessed using the concordance index (c-index). The predicted and actual survival was evaluated through calibration plots.

Results: The c-index of the nomogram was $0.779(95 \% \mathrm{Cl}, 0.659-0.898)$. The predicted and actual survival probabilities are in good agreement in all calibration curve.

Conclusion: This study built the first survival nomogram for patients with surgical resected RLMS.

\section{Introduction}

Leiomyosarcoma (LMS) is a mesenchymal malignant tumor with distinct smoothmuscle differentiation[1]. accounting for about 7-10\% of all soft tissue sarcoma (STS) and is the one of the most common STS subtype[2]. Currently, surgery is the best treatment for LMS that provides cure. But about $70 \%$ patients suffer from disease recurrence within five years after surgery[3].

Nomograms are a statistical tool used to predict an individual's prognosis and have been widely applied in LMS[4-6]. But French Sarcoma Group's reported that, LMS represent a heterogeneous group of tumors, and RLMS having totally different clinical outcome and molecular features than others[7]. Though it has been reported that the tumor size, resection margin status and tumor grade are related risk factors for the prognosis of retroperitoneal leiomyosarcoma[8-10]. However, as far as we know, there is currently no nomogram which constructed to predict the survival of RLMS patients only.

Therefore the aims of this study were (1) to investigate the independent prognostic factors for the overall survival (OS) of RLMS patients and after surgical resection (2) to construct a nomogram to predict the 1 year, 2 years and 5years OS of individual RLMS patients.

\section{Patients \& Methods}

\section{Patients}

The study was approved by the Ethics Committee of Shanghai Public Health Clinical Center. All enrolled patients signed an informed consent form for data collection during their hospitalization. 118 consecutive patients who undergone abdominal surgery only between September 2010 to December 2020 at the Sarcoma Center, General Surgery Department, Shanghai Public Health Clinical Center, Fudan University, Shanghai, China were included. The inclusion criteria were as follows: (a) complete clinical information, (b) complete follow-up information, (c) histologically confirmed leiomyosarcoma, (d) the 
primary site located in the retroperitoneum. Leiomyosarcoma is defined by the characteristic pathological features of H\&E staining, including oval or cigar-shaped nuclei with blunt ends, variable eosinophilic cytoplasm, and uniform a-sma, desmin, and/or h-caldesmon positivity staining, and all GISTs (CD117+ and CD34+) were excluded[11].

Patient and tumor variables, included gender, age ( $<60$ or $\geq 60)$, metastatic disease (patients with concurrent distant metastases or not), times of surgery (initial, second or more), complete resection (complete: R0, negative microscopic margin; incomplete : R1, positive microscopic margin or R2, macroscopically incomplete), number of resected organ (0-1 or $>1$ ), tumor burden (the sum of the largest diameters of all tumors), Federation Nationale des Centres de Lutte Contre le Cancer (FNCLCC) grade[12], multifocality, Inferior vena cava invasion, radiation (including preoperative and postoperative), chemotherapy (including preoperative and postoperative), other therapy (intervention, radiofrequency, gamma knife and intraperitoneal hyperthermic perfusion chemotherapy). The treatment plan for all patients is determined by a multidisciplinary team, including specialists in surgical oncology, medical oncology, radiation oncology, and pathology. Adjuvant treatments were used on a case-by-case basis. Each postoperative follow-up requires clinical and CT or MRI imaging of the chest, abdomen and pelvis. The initial follow-up is every 3-4 months, then every 6 months after 2 years, and every year after 5 years.

Statistical analysis

Statistical analyses were performed with SPSS version 22.0 (Chicago, IL, USA) and R software (version 3.1.1; http://www.r-project.org/) with the "rms", "foreign", and "survival" packages. The primary end-point of the study was OS (defined as the time from date of the surgery to the time of death or last contact). OS was estimated by the Kaplan-Meier method. The independent risk factors for OS were determined by Cox regression model. Variables with $\mathrm{p}$-values $<0.2$ in univariate analysis were included in multivariate analysis. Then variables in the multivariate analysis with a $p$ value $<0.1$ were used to construct the nomogram to predict 1-,2- and 5-year OS after surgery. The predictive accuracy of the nomogram was assessed by calculating the c-index and drawing a calibration curve to judge the discriminative ability. Then internally validated by Bootstrap method. Finally, the cohort was divided into low-risk group and high-risk group based on the median score calculated by the nomogram. The OS of two group was estimated by the Kaplan-Meier method, and the difference was compared with the log-rank test. All the tests were two-tailed and $\mathrm{P}<0.05$ considered statistically significance.

\section{Results}

Demographics and clinical characteristics of 118 patients are provided in Table 1. Most patients are female ( $n=99,83.9 \%)$, no distant metastasis when visiting our center $(n=106,89.8 \%)$, and the tumor was completely resected $(n=106,89.8 \%)$. Patients with the initial operation, the second operation and undergone more than twice operations accounted for $39.8 \%(n=47), 34.7 \%(n=41)$ and $25.4 \%(n=30)$, respectively. The proportions of FNCLCC classification are equal as grade 1, grade 2 and grade level 3 $(31.4 \%, 30.5 \%$ and $38.1 \%$, respectively). $73.7 \%(n=85)$ of patients had tumor burden greater than $5 \mathrm{~cm}$, 
23.7\% $(n=28)$ of patients had multifocal disease, and $55.1 \%(n=65)$ of patients had more than one organ removed. In terms of adjuvant therapy, $18.6 \%(n=22)$ patients received radiotherapy, $39 \%(n=46)$ patients received chemotherapy, and $12.7 \%(n=15)$ patients received other treatments.

Survival analysis

The median OS (Figure 1) for all patients was 47.8 (95\% confidence interval [Cl], 35.9-59.7) months. The median follow-up for the surviving patients was 31 (range, 4-113) months. The 5-year OS rate was $44.7 \%$ $(95 \% \mathrm{Cl}, 32.6 \%-56.8 \%)$.

In the univariable analysis, variables including number of resected organ $(p=0.012)$, tumor burden $(p=0.005)$ and FNCLCC grade $(p<0.001)$ were related to OS (Table 2$)$. Variables with $p<0.2$ in the univariate analysis were further included into the multivariate analysis. Multivariate analysis suggests that FNCLCC grade ( $p<0.001$, Grade 2 vs. Grade 1 [HR=3.320, 95\% Cl 1.088-10.129], Grade 3 vs. Grade 1 [HR=11.693, 95\% Cl 4.183-32.689]) and multifocal disease ( $p=0.025, \mathrm{HR}=2.333,95 \% \mathrm{Cl} 1.114-4.887)$ are independent risk factors for OS (Table 2).

Nomogram development and validation

Variables with $p<0.1$ in multivariate analysis (number of resected organ, tumor burden, FNCLCC grade and multifocal disease) were used to construct the OS nomogram for patients with RLMS who had the abdominal lesions removed (Figure 2).

The c-index after internal validation was $0.779(95 \% \mathrm{Cl}, 0.659-0.898)$. The calibration curves of 1-year, 2year, and 5-year OS probabilities show the predicted and actual survival probabilities are in good agreement (Figure 3).

The nomogram gives a specific value to quantify each variable. According to the total score, 118 patients were divided into low-risk group ( $<100)$ and high-risk group $(\geq 100)$. The median OS was $116.5(95 \% \mathrm{Cl},-)$ and 30.0 ( $95 \% \mathrm{Cl}, 24.2-35.8$ months) months, respectively. There was a statistically significant difference between the two groups $(p<0.001)$ (Figure 4$)$.

\section{Discussion}

Since the statistical prediction model can be simplified into a continuous numerical estimate tailored to individual patient conditions, the role of the nomogram prediction model has gradually become prominent in the era of precision medicine. In order to improve the estimation of the prognosis for patients with subtype pathology, some nomogram prediction models have been incorporated into the AJCC staging system. In the eighth edition of the AJCC manual, the nomogram by Trans-Atlantic Retroperitoneal Sarcoma Working Group for retroperitoneal soft tissue sarcoma (RPS) [13] was included as a model that met all AJCC quality criteria. 
There have been many reports on nomogram prediction models for leiomyosarcoma. For example, MingFeng Xue et al. used the SEER database to establish a nomogram prediction model based on 1528 patients with extremity leiomyosarcoma, which can predict 5- and 10-year OS and cancer specific survival, and The $\mathrm{C}$-index values for internal validation of $\mathrm{OS}$ and cancer specific survival prediction were 0.776 (95\% $\mathrm{Cl} 0.752-0.801)$ and 0.835 (95\% $\mathrm{Cl} 0.810-0.860)$, respectively[4]. Oliver Zivanovic et al. used a cohort of 185 cases of uterine leiomyosarcoma to establish a nomogram prediction model that can predict 5-year OS, with a c-index of 0.67 (95\% confidence interval, 0.63-0.72)[6]. However, not only specific STS histology but also the site of origin maters in determining outcomes in patients with soft tissue sarcoma. But there is currently no nomogram prediction model focused on retroperitoneal leiomyosarcoma only. Therefor, we developed and internally validated a novel, RLMS-specific nomogram for predicting 1-, 2- and 5-year OS, and the c-index of our nomogram was 0.779 ( $95 \% \mathrm{Cl}, 0.659-0.898)$, the calibration plots shows that the predicted OS rate was perfectly match with the actual OS rate.

RLMS is a very rare mesenchymal malignant that originates in the retroperitoneal space. Its incidence rate is less than 1 per million population[2]. For this usually fatal but rare disease, the question of how to obtain long-term survival is very important. Because of metastasis, multifocal disease, and multiple organ resection are usually contraindications to surgery, the potential beneficial effects of surgery are not always obvious, which lead decision-making very complicated. Because of metastasis, multifocal tumor, and multiple organ resection are usually contraindications to surgery[14]. And it is extremely difficult for clinical decision-making whether or not patients with recurrence should undergo surgery. However, we can get some hints in the nomogram established in this research. For example, for those diseases where the preoperative assessment does not require combined organ resection, tumor burden is less than $5 \mathrm{~cm}$, FNCLCC grade 1 and single center disease, even if it is a multiple recurrence disease, the 5-year OS rate after may exceed $90 \%$, and the operation is obvious profitable. On the contrary, those who need combined resection of multiple organs, tumor burden is greater than $5 \mathrm{~cm}$, FNCLCC grade 3 , multifocal disease, the nomogram score is greater than 200 points, and the OS is less than $30 \%$ at 2 years after surgery. For such people, the choice of surgery needs to be more cautious.

In the survival analysis, we found that FNCLCC grade and multifocal disease are independent risk factors for postoperative OS. The FNCLCC system is a commonly used histological grading system for soft tissue sarcomas, and it is one of the best indicators for predicting metastasis-free survival and 0S[15]. This study is similar to the previous study reported by Qian Li et al., for RLMS patients with recurrent or metastatic disease who had a higher FNCLCC grade experienced worse prognosis[16]. Consistent with previous reports on RPS, patients with multifocal disease accounted for $23 \%$ of patients in this study[17], and compared with single-center disease, the risk of death in patients with multifocal disease increased by two times. Although there have been reports about the role of multifocal disease in the prognosis of RPS, as far as we know, this research is the first to report its a independent prognostic factors in RLMS.

This study had certain limitations. First, this study was based on a retrospective cohort. The selection and inclusion of retrospective patients may cause research bias. Second, the median follow-up time for surviving patients in this study was 31 months, and further extension of the follow-up time will increase 
the reliability of the data. Third, although internal verification shows that this nomogram was a good predictive model, it still needs to further incorporate data from other centers for external verification.

\section{Conclusions}

This study offers a RLMS-specific postoperative nomogram to assess OS for RLMS patients after surgery. It improves current RLMS staging with divided patients into low-risk and high-risk group. With the advantage of focusing on the RLMS, this tool can assist doctors in clinical decision-making.

\section{Abbreviations}

RLMS retroperitoneal leiomyosarcoma

c-index concordance index

LMS leiomyosarcoma

STS soft tissue sarcoma

FNCLCC Federation Nationale des Centres de Lutte Contre le Cancer

OS overall survival

RPS retroperitoneal soft tissue sarcoma

\section{Declarations}

Acknowledgements

We would like to thank our patients, without whom this study would not possible.

Authors' contributions

$A B Z$ and WQL developed the concept of the article. ABZ and YF developed the design and methodology. LJM contributed to the manuscript revision. HXT and YZ contributed to the drafting of the manuscript. All authors read and approved the final manuscript.

Funding

No funding.

Availability of data and materials

The datasets used and/or analyzed during the current study are available from the corresponding author on reasonable request. 
Ethics approval and consent to participate

The study was approved by the Ethics Committee of Shanghai Public Health Clinical Center. All enrolled patients signed an informed consent form for data collection during their hospitalization.

Consent for publication

Not applicable.

Competing interests

The authors declare that they have no competing interests.

\section{References}

1. Fletcher CDM, Unni KK, Mertens F (2002) World Health Organization Classification of tumors: pathology and genetics of tumors of soft tissue and bone. IARC Press, Lyon.

2. Bathan AJ, Constantinidou A, Pollack SM, Jones RL. Diagnosis, prognosis, and management of leiomyosarcoma: recognition of anatomic variants. CURR OPIN ONCOL, 25(4), 384-389 (2013).

3. Ikoma N, Torres KE, Lin HY et al.. Recurrence patterns of retroperitoneal leiomyosarcoma and impact of salvage surgery. J SURG ONCOL, 116(3), 313-319 (2017).

4. Xue M, Chen G, Dai J, Hu J. Development and Validation of a Prognostic Nomogram for Extremity Soft Tissue Leiomyosarcoma. FRONT ONCOL, 9, 346 (2019).

5. lasonos $A$, Keung EZ, Zivanovic $O$ et al.. External validation of a prognostic nomogram for overall survival in women with uterine leiomyosarcoma. CANCER-AM CANCER SOC, 119(10), 1816-1822 (2013).

6. Lu YJ, Wang H, Fang LY et al.. A nomogram for predicting overall survival in patients with uterine leiomyosarcoma: a SEER population-based study. FUTURE ONCOL, 16(10), 573-584 (2020).

7. Italiano A, Lagarde P, Brulard C et al.. Genetic profiling identifies two classes of soft-tissue leiomyosarcomas with distinct clinical characteristics. CLIN CANCER RES, 19(5), 1190-1196 (2013).

8. Kim HJ, Cho YJ, Kim SH et al.. Leiomyosarcoma: investigation of prognostic factors for riskstratification model. INT J CLIN ONCOL, 20(6), 1226-1232 (2015).

9. Abraham JA, Weaver MJ, Hornick JL, Zurakowski D, Ready JE. Outcomes and prognostic factors for a consecutive case series of 115 patients with somatic leiomyosarcoma. J BONE JOINT SURG AM, 94(8), 736-744 (2012).

10. Gronchi A, Miceli R, Allard MA et al.. Personalizing the approach to retroperitoneal soft tissue sarcoma: histology-specific patterns of failure and postrelapse outcome after primary extended 
resection. ANN SURG ONCOL, 22(5), 1447-1454 (2015).

11. Katz SC, DeMatteo RP. Gastrointestinal stromal tumors and leiomyosarcomas. J SURG ONCOL, 97(4), 350-359 (2008).

12. Coindre JM. Grading of soft tissue sarcomas: review and update. ARCH PATHOL LAB MED, 130(10), 1448-1453 (2006).

13. Gronchi A, Miceli R, Shurell E et al.. Outcome prediction in primary resected retroperitoneal soft tissue sarcoma: histology-specific overall survival and disease-free survival nomograms built on major sarcoma center data sets. J CLIN ONCOL, 31(13), 1649-1655 (2013).

14. van Houdt WJ, Fiore M, Barretta F et al.. Patterns of recurrence and survival probability after second recurrence of retroperitoneal sarcoma: A study from TARPSWG. CANCER-AM CANCER SOC, 126(22), 4917-4925 (2020).

15. Coindre JM, Terrier P, Guillou $L$ et al.. Predictive value of grade for metastasis development in the main histologic types of adult soft tissue sarcomas: a study of 1240 patients from the French Federation of Cancer Centers Sarcoma Group. CANCER-AM CANCER SOC, 91(10), 1914-1926 (2001).

16. Li Q, Zhuang R, Zhu J et al.. Prognostic factors in patients with recurrent or metastatic retroperitoneal leiomyosarcoma. FUTURE ONCOL, 11(12), 1759-1766 (2015).

17. Anaya DA, Lahat G, Liu J et al.. Multifocality in retroperitoneal sarcoma: a prognostic factor critical to surgical decision-making. ANN SURG, 249(1), 137-142 (2009).

\section{Tables}

Table 1 Demographics and clinical characteristics of 118 patients with retroperitoneal leiomyosarcoma 


\begin{tabular}{|c|c|c|}
\hline Characteristics & $\mathrm{N}(\mathrm{N}=118)$ & $\%$ of Total \\
\hline \multicolumn{3}{|l|}{ Gender } \\
\hline Male & 19 & 16.1 \\
\hline Female & 99 & 83.9 \\
\hline \multicolumn{3}{|l|}{ Age, years } \\
\hline$<60$ & 76 & 64.4 \\
\hline$\geq 60$ & 42 & 35.6 \\
\hline \multicolumn{3}{|l|}{ Metastatic disease } \\
\hline Yes & 12 & 10.2 \\
\hline No & 106 & 89.8 \\
\hline \multicolumn{3}{|l|}{ Times of surgery } \\
\hline Initial & 47 & 39.8 \\
\hline Second & 41 & 34.7 \\
\hline More than twice & 30 & 25.4 \\
\hline \multicolumn{3}{|l|}{ Complete resection } \\
\hline Yes & 106 & 89.8 \\
\hline No & 12 & 10.2 \\
\hline \multicolumn{3}{|c|}{ Number of resected organ } \\
\hline $0-1$ & 53 & 44.9 \\
\hline$>1$ & 65 & 55.1 \\
\hline \multicolumn{3}{|l|}{ Tumor burden, cm } \\
\hline$\leq 5$ & 31 & 26.3 \\
\hline$>5$ & 85 & 73.7 \\
\hline \multicolumn{3}{|l|}{ FNCLCC } \\
\hline Grade 1 & 37 & 31.4 \\
\hline Grade 2 & 36 & 30.5 \\
\hline Grade 3 & 45 & 38.1 \\
\hline \multicolumn{3}{|l|}{ Multifocal disease } \\
\hline Yes & 28 & 23.7 \\
\hline
\end{tabular}




\begin{tabular}{lcc} 
No & 90 & 76.3 \\
\hline Inferior vena cava invasion & & \\
\hline Yes & 17 & 14.4 \\
\hline No & 101 & 85.6 \\
\hline Radiation & & \\
\hline Yes & 22 & 18.6 \\
\hline No & 96 & 81.4 \\
\hline Chemotherapy & & \\
\hline Yes & 46 & 39.0 \\
\hline No & 72 & 61.0 \\
\hline Other therapy & & 12.7 \\
\hline Yes & 15 & 87.3 \\
\hline No & 103 & \\
\hline
\end{tabular}

Table 2 Univariable and multivariable analyses to determine independent predictors of overall survival of retroperitoneal leiomyosarcoma 


\begin{tabular}{|c|c|c|c|c|}
\hline \multirow[t]{2}{*}{ Variables } & \multicolumn{2}{|c|}{ Univariate analysis } & \multicolumn{2}{|c|}{ Multivariate analysis } \\
\hline & $\begin{array}{l}\text { Hazard Ratio } \\
\text { } 95 \% \mathrm{Cl} \otimes\end{array}$ & $P$ value & $\begin{array}{l}\text { Hazard Ratio } \\
\qquad 95 \% \mathrm{Cl} \otimes\end{array}$ & $P$ value \\
\hline Gender female vs. male & $\begin{array}{l}1.006(0.672- \\
1.507)\end{array}$ & 0.975 & & \\
\hline Age $\geq 60$ vs. $<60$ & $\begin{array}{l}1.242(0.686- \\
2.248)\end{array}$ & 0.475 & & \\
\hline Metastatic disease yes vs. no & $\begin{array}{l}1.929(0.814- \\
4.570)\end{array}$ & 0.135 & $\begin{array}{l}1.925(0.740- \\
5 .-12)\end{array}$ & 0.180 \\
\hline Times of surgery & & 0.486 & & \\
\hline second vs. first & $\begin{array}{l}0.664(0.320- \\
1.379)\end{array}$ & 0.272 & & \\
\hline more than twice vs. first & $\begin{array}{l}1.020(0.510- \\
2.040)\end{array}$ & 0.955 & & \\
\hline Complete resection no vs. yes & $\begin{array}{l}1.303(0.846- \\
2.007)\end{array}$ & 0.230 & & \\
\hline Number of resected organ $>1$ vs. $0-1$ & $\begin{array}{l}2.173(1.187- \\
3.981)\end{array}$ & 0.012 & $\begin{array}{l}1.797(0.909- \\
3.552)\end{array}$ & 0.092 \\
\hline Tumor burden $>5$ vs. $\leq 5$ & $\begin{array}{l}5.351(1.655- \\
17.299)\end{array}$ & 0.005 & $\begin{array}{l}2.945(0.861- \\
10.070)\end{array}$ & 0.085 \\
\hline FNCLCC & & $<0.001$ & & $<0.001$ \\
\hline Grade 2 vs. Grade 1 & $\begin{array}{l}3.352 \text { (1.137- } \\
9.880)\end{array}$ & 0.028 & $\begin{array}{l}3.320(1.088- \\
10.129)\end{array}$ & 0.035 \\
\hline Grade 3 vs. Grade 1 & $\begin{array}{l}9.355(3.560- \\
24.589)\end{array}$ & $<0.001$ & $\begin{array}{l}11.693(4.183- \\
32.689)\end{array}$ & $<0.001$ \\
\hline Multifocal disease yes vs. no & $\begin{array}{l}1.906(0.995- \\
3.651)\end{array}$ & 0.052 & $\begin{array}{l}2.333(1.114- \\
4.887)\end{array}$ & 0.025 \\
\hline Inferior vena cava invasion yes vs. no & $\begin{array}{l}1.259(0.753- \\
2.104)\end{array}$ & 0.380 & & \\
\hline Radiation yes vs. no & $\begin{array}{l}1.767(0.786- \\
3.970)\end{array}$ & 0.168 & $\begin{array}{l}1.400(0.580- \\
3.383)\end{array}$ & 0.454 \\
\hline Chemotherapy yes vs. no & $\begin{array}{l}1.005(0.548- \\
1.842)\end{array}$ & 0.987 & & \\
\hline Other therapys yes vs. no & $\begin{array}{l}1.005(0.395- \\
2.555)\end{array}$ & 0.992 & & \\
\hline
\end{tabular}

\section{Figures}




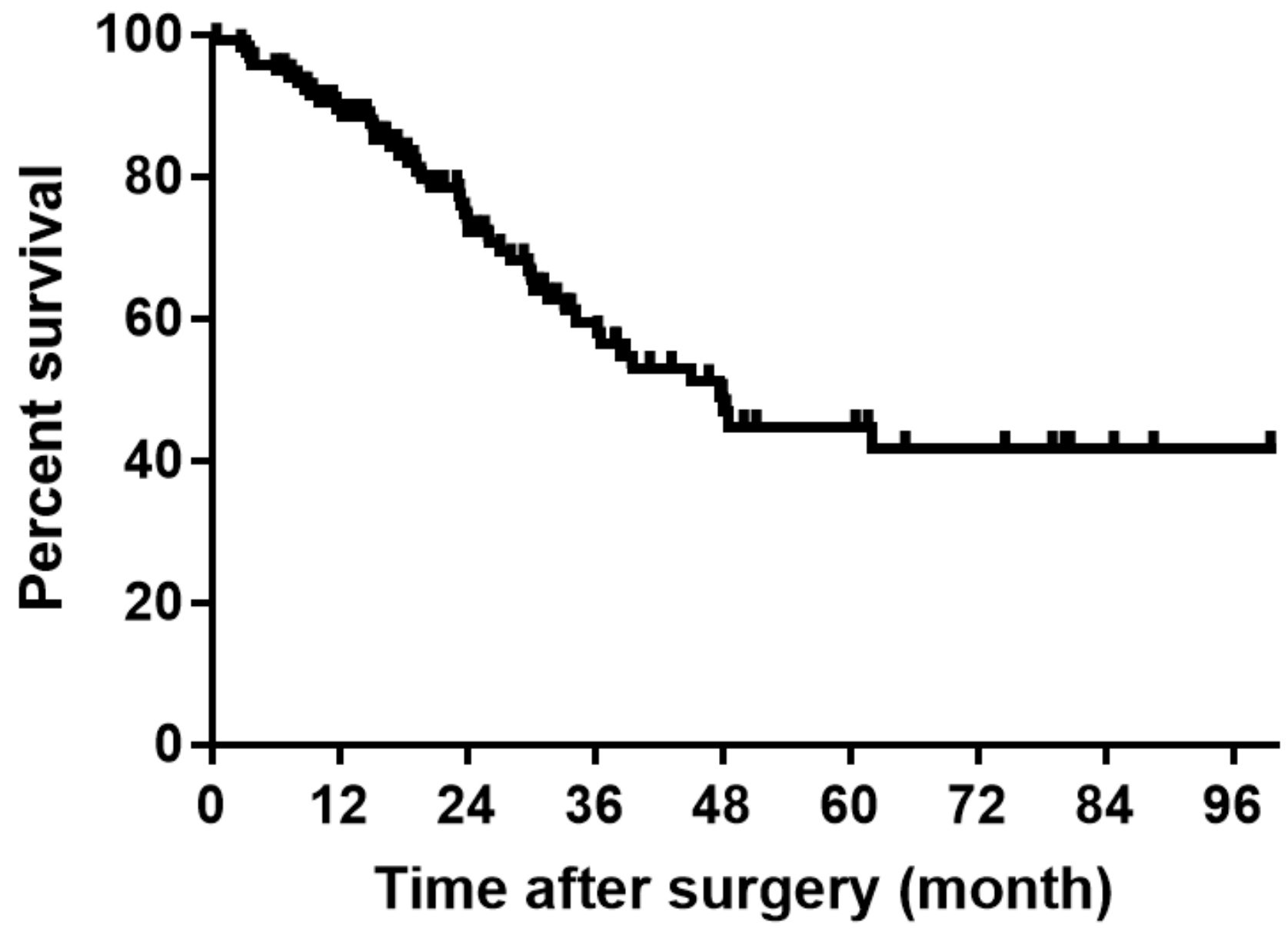

Figure 1

Overall survival in patients with retroperitoneal leiomyosarcoma 
Points

Resected_organ

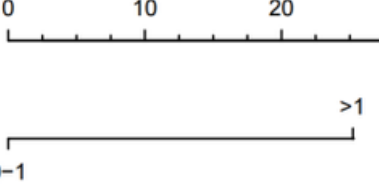

Tumor_size

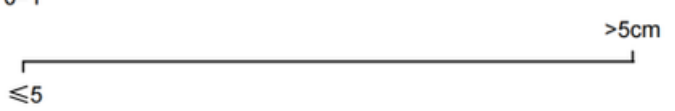

FNCLCC_grade

Multifocal_disease

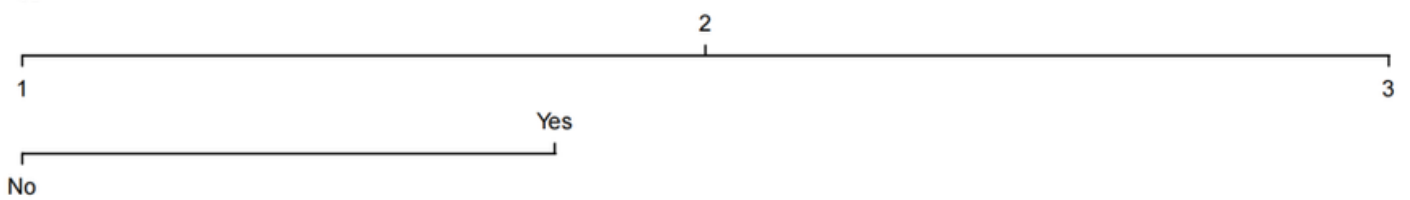

Total Points

\begin{tabular}{llllllllllll}
\hline 0 & 20 & 40 & 60 & 80 & 100 & 120 & 140 & 160 & 180 & 200 & 220
\end{tabular}

1 year OS rate

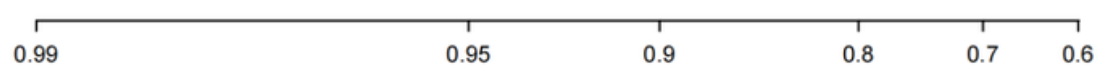

2 years OS rate

\begin{tabular}{|c|c|c|c|c|c|c|c|}
\hline 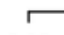 & 1 & 1 & 1 & 1 & 1 & 1 & 1 \\
\hline 0.99 & 0.95 & 0.9 & 0.8 & 0.7 & 0.6 & 0.5 & 0.4 \\
\hline
\end{tabular}

5 years OS rate

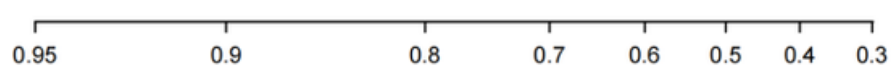

Figure 2

Nomogram for 1-year, 2-year and 5-year overall survival in patients with retroperitoneal leiomyosarcoma
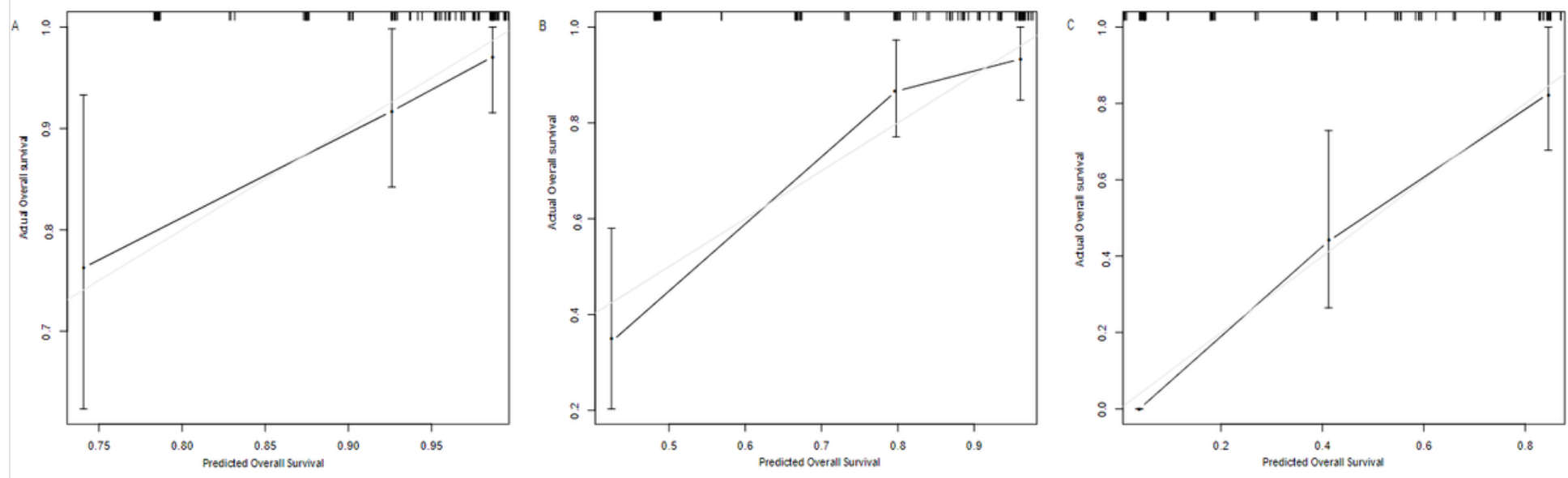

Figure 3

Calibration plots for internal validation of (A) 1-, (B) 2- and (C) 5-year overall survival nomogram. 


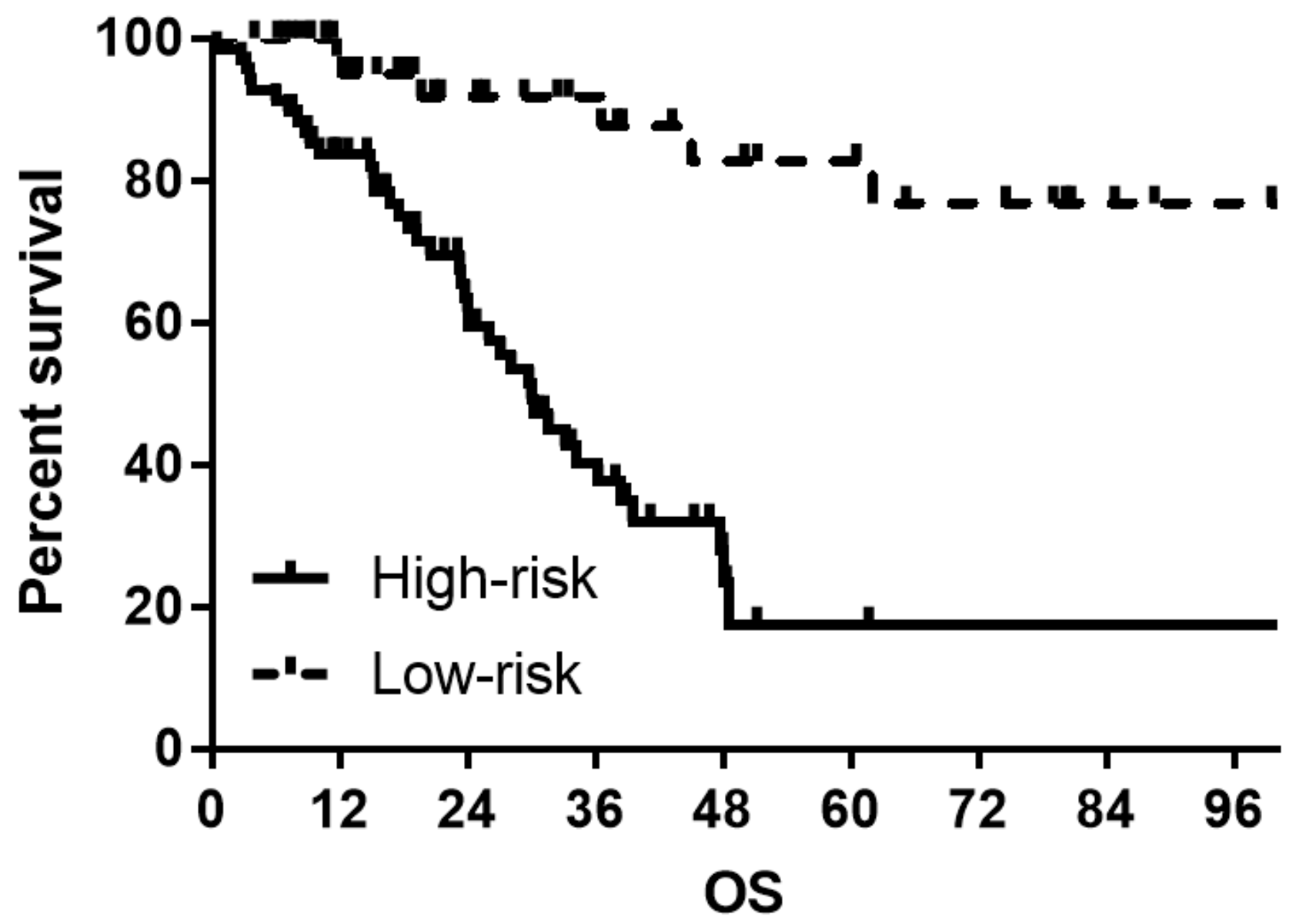

Figure 4

OS curves stratified by the score calculated by the nomogram and was stratified according to the risk score as follows: low-risk group (<100) and high-risk group $(\geq 100)$ 